\title{
Are there approximate relations among transverse momentum dependent distribution functions?
}

\author{
H. Avakian, ${ }^{1}$ A. V. Efremov ${ }^{2}$ K. Goeke, ${ }^{3}$ A. Metz,${ }^{3,4}$ P. Schweitzer,${ }^{3}$ and T. Teckentrup ${ }^{3}$ \\ ${ }^{1}$ Thomas Jefferson National Accelerator Facility, Newport News, VA 23606, U.S.A. \\ ${ }^{2}$ Joint Institute for Nuclear Research, Dubna, 141980 Russia \\ ${ }^{3}$ Institut für Theoretische Physik II, Ruhr-Universität Bochum, D-44780 Bochum, Germany \\ ${ }^{4}$ Department of Physics, Barton Hall, Temple University, Philadelphia, PA 19122-6082, U.S.A.
}

(Dated: September 2007)

\begin{abstract}
Certain exact relations among transverse momentum dependent parton distribution functions due to QCD equations of motion turn into approximate ones upon the neglect of pure twist-3 terms. On the basis of available data from HERMES we test the practical usefulness of one such "WandzuraWilczek-type approximation", namely of that connecting $h_{1 L}^{\perp(1) a}(x)$ to $h_{L}^{a}(x)$, and discuss how it can be further tested by future CLAS and COMPASS data.
\end{abstract}

PACS numbers: 13.88.+e, 13.85.Ni, 13.60.-r, 13.85.Qk

Keywords: Semi-inclusive deep inelastic scattering, tranverse momentum dependent distribution functions, single spin asymmetry, Wandzura-Wilczek approximation

\section{INTRODUCTION}

Semi-inclusive deep inelastic lepton nucleon scattering (SIDIS), hadron production in electron-positron annihilations and the Drell-Yan process [1, 2, 3, 4, 5, 6, 7, $8,9,10,11,12,13,14$ allow to access information on transverse momentum dependent (TMD) parton distribution functions (pdf) and fragmentation functions [15]. In order to be sensitive to "intrinsic" transverse parton momenta it is necessary to measure adequate transverse momenta in the final state, e.g. in SIDIS the transverse momenta of produced hadrons with respect to the virtual photon. Some data on such processes are available $\sqrt{16}, 17,18,19,20,21,22,23,24,25,26,22,28,29,30$, [31, 32], and at least in the case of twist-2 observables factorization applies $33,34,35$.

Eight twist-2 and sixteen twist-3 TMD pdfs describe the nucleon structure in these processes, namely [36, 37]

$$
\underbrace{f_{1}^{a}, f_{1 T}^{\perp a}, g_{1 L}^{a}, g_{1 T}^{a}, h_{1 T}^{a}, h_{1 L}^{\perp a}, h_{1 T}^{\perp a}, h_{1}^{\perp a}}_{\text {twist-2 }} \underbrace{e^{a}, g_{T}^{a}, h_{L}^{a}, \ldots}_{\text {twist-3 }}
$$

which are functions of $x$ and $\mathbf{p}_{T}^{2}$. (The dots denote thirteen further twist-3 TMD pdfs. The renormalization scale dependence is not indicated for brevity.) Integrating over transverse momenta one is left with six independent "collinear" pdfs [38, 39]

$$
\underbrace{f_{1}^{a}(x), \quad g_{1}^{a}(x), \quad h_{1}^{a}(x)}_{\text {twist-2 }}, \underbrace{e^{a}(x), \quad g_{T}^{a}(x), \quad h_{L}^{a}(x)}_{\text {twist-3 }} .
$$

where the relations hold $j(x)=\int \mathrm{d}^{2} \mathbf{p}_{T} j\left(x, \mathbf{p}_{T}^{2}\right)$ for $j=f_{1}^{a}, e^{a}, g_{T}, h_{L}$ while $g_{1}^{a}(x)=\int \mathrm{d}^{2} \mathbf{p}_{T} g_{1 L}^{a}\left(x, \mathbf{p}_{T}^{2}\right)$ and $h_{1}^{a}(x)=\int \mathrm{d}^{2} \mathbf{p}_{T}\left\{h_{1 T}^{a}\left(x, \mathbf{p}_{T}^{2}\right)+\mathbf{p}_{T}^{2} /\left(2 M_{N}^{2}\right) h_{1 T}^{\perp a}\left(x, \mathbf{p}_{T}^{2}\right)\right\}$.

In view of the prolification of novel functions in (11) one may ask whether some of the unknown TMD pdfs could be related to (possibly better) known ones. Since all structures in (11) are independent [36], any such relations can only be approximate.
Candidates for such approximate relations can be obtained as follows. From QCD equations of motion (eom), one obtains among others the following exact relations [7]

$$
\begin{aligned}
& g_{1 T}^{\perp(1) a}(x) \stackrel{\text { eom }}{=} x g_{T}^{a}(x)-x \tilde{g}_{T}^{a}(x), \\
&-2 h_{1 L}^{\perp(1) a}(x) \stackrel{\text { eom }}{=} x h_{L}^{a}(x)-x \tilde{h}_{L}^{a}(x),
\end{aligned}
$$

with the transverse moments defined as $\left(g_{1 T}^{\perp(1)}\right.$ analog)

$$
h_{1 L}^{\perp(1) a}(x) \equiv \int \mathrm{d}^{2} \mathbf{p}_{T} \frac{\mathbf{p}_{T}^{2}}{2 M_{N}^{2}} h_{1 L}^{\perp a}\left(x, \mathbf{p}_{T}^{2}\right),
$$

and with $\tilde{g}_{T}^{a}(x), \tilde{h}_{L}^{a}(x)$ denoting pure twist-3 "interaction dependent" terms due to quark-gluon-quark correlations (and current quark mass terms). In the next step, we recall the relations among the collinear pdfs (2) 39, 40, 41]

$$
\begin{array}{r}
g_{T}^{a}(x)=\int_{x}^{1} \frac{\mathrm{d} y}{y} g_{1}^{a}(y)+\tilde{g}_{T}^{a}(x), \\
h_{L}^{a}(x)=2 x \int_{x}^{1} \frac{\mathrm{d} y}{y^{2}} h_{1}^{a}(y)+\tilde{h}_{L}^{\prime a}(x),
\end{array}
$$

where $\tilde{g}_{T}^{\prime a}(x), \tilde{h}_{L}^{\prime a}(x)$ also denote pure twist-3 (and mass) terms [42, 43], though different ones than in (3, 44). Eqs. (6, 76) isolate "pure twist-3 terms" in the "twist-3" pdfs $g_{T}^{a}(x), h_{L}^{a}(x)$. This is because in (2) the underlying "working definition" of twist [44] (a pdf is "twist $t$ " if its contribution to the cross section is suppressed, in addition to kinematic factors, by $1 / Q^{t-2}$ with $Q$ the hard scale in the process) differs from the strict definition of twist (mass dimension of the operator minus its spin).

The remarkable observation is that $\tilde{g}_{T}^{\prime a}(x)$ is consistent with zero within error bars [45, 46, 47, 48, 49] and to a good accuracy

$$
g_{T}^{a}(x) \stackrel{\mathrm{WW}}{\approx} \int_{x}^{1} \frac{\mathrm{d} y}{y} g_{1}^{a}(y) \quad \text { (exp. observation) }
$$


which is the "Wandzura-Wilczek (WW) approximation". Lattice QCD 50, 51] and the instanton model of the QCD vacuum [52] support this observation. Interestingly the latter predicts also $\tilde{h}_{L}^{\prime a}(x)$ to be small [53], such that

$$
h_{L}^{a}(x) \approx 2 x \int_{x}^{1} \frac{\mathrm{d} y}{y^{2}} h_{1}^{a}(y) \quad \text { (prediction). }
$$

On the basis of this positive experimental and (or) theoretical experience with the smallness of pure twist-3 (and mass) terms one may suspect that the analog terms in the relations (33 4) could also be negligible. If true one would have valuable $\mathrm{WW}$-type approximations

$$
\begin{aligned}
& g_{1 T}^{\perp(1) a}(x) \stackrel{! ?}{\approx} x \int_{x}^{1} \frac{\mathrm{d} y}{y} g_{1}^{a}(y), \\
& h_{1 L}^{\perp(1) a}(x) \stackrel{! ?}{\approx}-x^{2} \int_{x}^{1} \frac{\mathrm{d} y}{y^{2}} h_{1}^{a}(y),
\end{aligned}
$$

that could be satisfied with an accuracy comparable to that of (8). This remains to be tested in experiment.

An immediate application (or test) for the relations (10, 11) is provided by the following single/double spin asymmetries (SSA/DSA) in SIDIS

$$
\begin{aligned}
A_{U L}^{\sin 2 \phi} & \propto \sum_{a} e_{a}^{2} h_{1 L}^{\perp(1) a} H_{1}^{\perp a}, \\
A_{L T}^{\cos \left(\phi-\phi_{S}\right)} & \propto \sum_{a} e_{a}^{2} g_{1 T}^{\perp(1) a} D_{1}^{a},
\end{aligned}
$$

where the first index $U$ (or $L$ ) means that the leptons are un- (or longitudinally) polarized, the second $L$ (or $T$ ) indicates the longitudinal (or transverse) polarization of the nucleon, and $\phi\left(\phi_{S}\right)$ denotes the azimuthal angle of the produced hadron $h$ (target polarization vector $S$ ) with respect to the axis defined by the virtual photon, see Fig. 2. The superscripts $\sin 2 \phi$ or $\cos \left(\phi-\phi_{S}\right)$ mean that the spin asymmetries were weighted correspondingly in order to isolate the contributions responsible for the particular azimuthal distributions.

In (12) $H_{1}^{\perp a}$ denotes the Collins fragmentation function [3, 4, 5] on which data from SIDIS [21, 22, 23, 24] on the SSA

$$
A_{U T}^{\sin \left(\phi+\phi_{S}\right)} \propto \sum_{a} e_{a}^{2} h_{1}^{a} H_{1}^{\perp a}
$$

and from $e^{+} e^{-}$annihilations [28, 29 give rise to a first but already consistent picture of $H_{1}^{\perp}[54,55,56]$. The $D_{1}^{a}$ in (13) is the unpolarized fragmentation function which enters, of course, also the respective denominators in 12 . 14) that are proportional to $\sum_{a} e_{a}^{2} f_{1}^{a} D_{1}^{a}$.

Final HERMES [17, 18, 19] and preliminary CLAS 25] data on (12) and preliminary COMPASS data 32] on (13) are available, such that first tests of the WW-type approximations (10, 11) are now or soon possible.

In this note we shall present a test of the approximation (11). Under the assumption that this approximation works, we shall see that it yields results for the SSA (12) compatible with HERMES data [17, 18, 19]. From another point of view our work provides a first independent cross check from SIDIS for the emerging picture of $H_{1}^{\perp}$ [54, [55, 56]. The SSA (12) was recently studied in [57].

A test of the approximation (10) was suggested in [58] along the lines of the study of the SSA (13) discussed previously also in [59].

Among the eight structure functions in SIDIS described in terms of twist-2 pdfs and fragmentation functions [37] the SSAs (12, 13) are the only ones, for which WW-type approximations could be of use. Exact eomrelations exist, in fact, for all eight twist-2 pdfs in (1). But the relations (3, (4) are special in that they connect the respective TMD pdfs, namely $g_{1 T}^{\perp}$ and $h_{1 L}^{\perp}$, to "collinear" twist-3 pdfs, namely $g_{T}$ and $h_{L}$. Those in turn are related to twist- 2 pdfs, $g_{1}$ and $h_{1}$, by means of (experimentally established or theoretically predicted) WW-approximations (8, 9).

Experiments may or may not confirm that the WWtype approximations (10, 11) work.

What would it mean if (10, 11) were found to be satisfied to within a very good accuracy? First, that would be of practical use for understanding and interpreting the first data 17, 18, 19, 20, 21, 22, 23, 24, 25, 26, 27, 28, 29, $30,31,32$. Second, it would call for theoretical explanations why pure twist- 3 terms should be small. (Only for the smallness of the "collinear" pure twist-3 terms in (88 91) lattice QCD [50, 51] and/or instanton vacuum [52, 53] provide explanations.)

What would it mean if (10, 111) were found to work poorly? This scenario would be equally interesting. In fact, all eight pdfs in (1) are independent structures, and any of them contains different type of information on the internal structure of the nucleon. The measurement of the complete set of all eighteen structure functions available in SIDIS [6] is therefore indispensable for our aim to learn more about the nucleon structure.

One type of information accessible in this way concerns effects related to the orbital motion of quarks, and in particular correlations of spin and transverse momentum of quarks which are dominated by valence quarks and hence play a more important role at large $x$. E.g. it was shown that spin-orbit correlations may lead to significant contribution to partonic momentum and helicity distributions [60] in large- $x$ limit. Spin-orbit correlations are presumably of similar importance for transversity, and crucial for $h_{1 L}^{\perp}$, which describes transversely polarized quarks in a longitudinally polarized nucleon, and is a measure for the correlation of the transverse spin and the transverse momentum of quarks.

This note is organized as follows. In Sec. II we estimate $h_{1 L}^{\perp}$ by means of the WW-type approximation (11) using various different models for $h_{1}$, and discuss modelindependent features of these estimates. In Sec. III we introduce notations and definitions. In Sec. IV we evaluate the SSA (12) in the WW-type approximation (11) and compare the results to available HERMES data [17, 18, 19]. In Secs. V and VI we discuss what can be 
learned from future measurements at CLAS, and COMPASS. Sec. VII contains the conclusions.

\section{WW-TYPE APPROXIMATION FOR $h_{1 L}^{\perp}$}

In order to model $h_{1 L}^{\perp(1) a}(x)$ by means of the WW-type approximation (11) one inevitably has to use, in addition, models for the transversity pdf. Fig. 11 shows four different models: saturation of the Soffer bound [61] at the low initial scale of the leading order parameterizations 62, 63] (choosing $h_{1}^{u}>0$ and $h_{1}^{d}<0$ ), the chiral quark soliton model ( $\chi \mathrm{QSM})$ [64], the non-relativistic
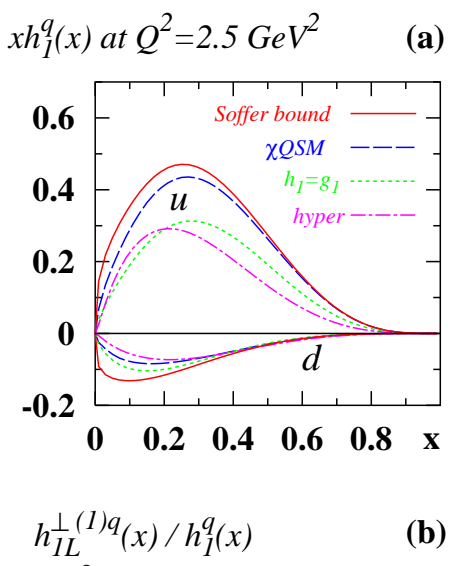

(b)
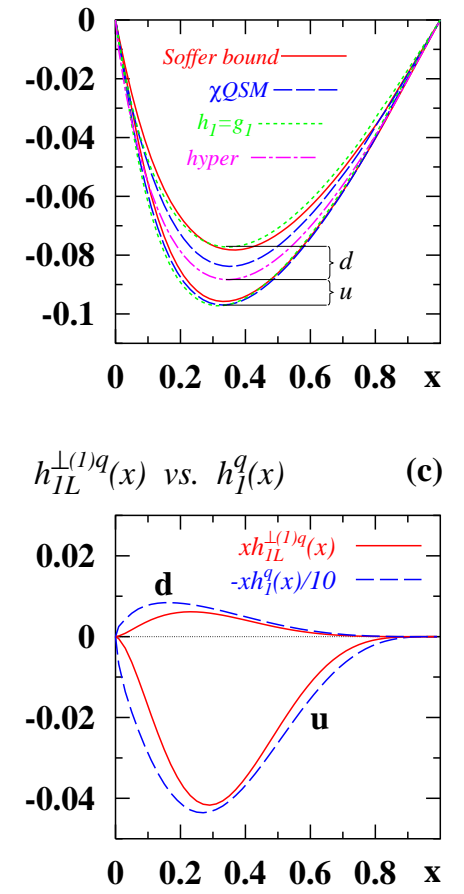

FIG. 1: (a) Transversity, $x h_{1}^{q}(x)$, vs. $x$, from various models. (b) The ratio $h_{1 L}^{\perp(1) q}(x) / h_{1}^{q}(x)$ vs. $x$ in various models, with $h_{1 L}^{\perp}$ estimated by means of the WW-type approximation (11). (c) $x h_{1 L}^{\perp(1) q}(x)$ vs. $x$ from the WW-type approximation (11) and $h_{1}^{a}(x)$ from $\chi$ QSM 64], in comparison with $\left(-\frac{1}{10}\right) x h_{1}^{q}(x)$ from that model. All results here refer to a scale of $2.5 \mathrm{GeV}^{2}$. model assumption $h_{1}^{a}(x)=g_{1}^{a}(x)$ at the low scale of the parameterization [63], and the hypercentral model [65]. All curves in Fig. 1 are leading-order evolved to $2.5 \mathrm{GeV}^{2}$ which is a relevant scale in experiment, see below.

These (and many other [66, 67]) models agree on that $h_{1}^{u}(x)>0$ and $h_{1}^{d}(x)<0$ with $\left|h_{1}^{d}(x)\right|<h_{1}^{u}(x)$, though the predictions differ concerning the magnitudes, see Fig. 17. Models in which antiquark distribution functions can be computed, e.g. 64, predict that the transversity antiquark pdfs are far smaller than the quark ones.

Let us therefore establish first a robust feature of the relation (11), namely the ratio $h_{1 L}^{\perp(1) q}(x) / h_{1}^{q}(x)$ exhibits little dependence on the transversity model, see Fig. 1]b.

A "universal" behaviour of this ratio at large $x$ is not surprizing. By inspecting (11) for large $x$ one finds

$$
\lim _{\text {large } x} \frac{h_{1 L}^{\perp(1) a}(x)}{h_{1}^{a}(x)} \sim(1-x),
$$

which agrees with general results from large- $x$ counting rules [68]. This is also true for (10). That the WW-type approximations respect the relative large- $x$ behaviour of the involved pdfs can intuitively be understood by considering that multi-parton-correlations are likely to vanish faster at large $x$ than twist- 2 terms.

Also a "universal" small- $x$ behaviour of the ratio can be understood from Eq. (11), namely for $h_{1}^{a}(x) \sim x^{\alpha}$ at small $x$ one obtains

$$
\lim _{\operatorname{small} x} \frac{h_{1 L}^{\perp(1) a}(x)}{h_{1}^{a}(x)} \sim \begin{cases}x & \text { for } \alpha \neq 1 \\ x \log x & \text { for } \alpha=1\end{cases}
$$

i.e. the ratio tends to zero with $x \rightarrow 0$ in any case. ${ }^{1}$

Nevertheless it is interesting to observe that the ratio is rather robust also at intermediate $x$. For the hypercentral model 65] the ratio is flavour-independent, since there $h_{1}^{u}(x)=-4 h_{1}^{d}(x)$ holds trivially due to the imposed $\mathrm{SU}(2)_{\text {spin }} \times \mathrm{SU}(2)_{\text {flavour }}$ spin-flavour-symmetry. In the other models one, however, observes departures from that, see Fig. 1b.

As a common feature we finally observe

$$
\left|\frac{h_{1 L}^{\perp(1) a}(x)}{h_{1}^{a}(x)}\right| \lesssim 0.1 .
$$

In the following we will use the $\chi$ QSM, see Fig. 11, which has several advantages. First, it is a faithful field theoretic model of the nucleon [72, 73] that describes the twist- 2 pdfs $f_{1}^{a}(x)$ and $g_{1}^{a}(x)$ within (10-30)\% accuracy 74. Second, this model is derived from the instanton vacuum model [75, 76] which predicts that the "collinear

\footnotetext{
1 Notice that all curves in Fig. 1 are results of leading order evolution 69] starting from low scales - ranging from $0.079 \mathrm{GeV}^{2}$ for 65], till $0.36 \mathrm{GeV}^{2}$ for $\chi \mathrm{QSM}$ [64]. Next-to-leading order evolution 70 and Regge asymptotics 71 predict a behaviour $h_{1}^{a}(x) \sim \mathcal{O}\left(x^{0}\right)$ for $x \rightarrow 0$.
} 
WW-type approximation" (9) works well 53. Third, below we will use $h_{1}^{a}(x)$ from the $\chi$ QSM in combination with information on the Collins effect from the analysis [55] where this model was used. This helps to minimize the model-dependence in our study. But we shall see that our conclusions do not depend on the choice of model.

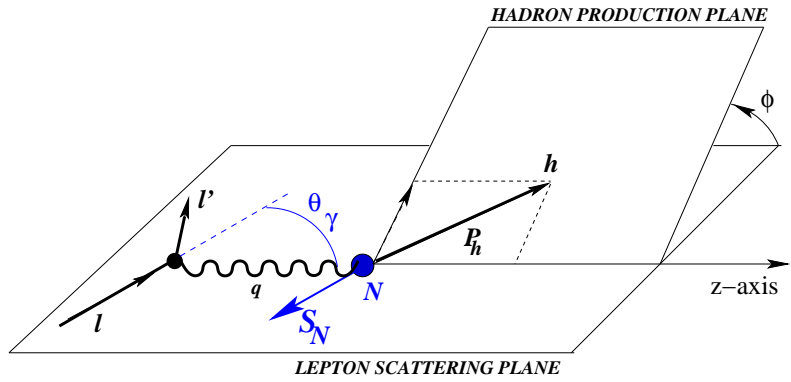

FIG. 2: Kinematics of the SIDIS process $l N \rightarrow l^{\prime} h X$ and the definitions of azimuthal angles in the lab frame. Here the target polarization is antiparallel to the beam (i.e. $\phi_{S}=\pi$ ).

\section{III. $A_{U L}^{\sin 2 \phi}$ AT HERMES}

Let us denote the momenta of the target, incoming and outgoing lepton by $P, l$ and $l^{\prime}$ and introduce $s=(P+l)^{2}$, the four-momentum transfer $q=l-l^{\prime}$ with $Q^{2}=-q^{2}$ and $W^{2}=(P+q)^{2}$. Then $y=P q / P l$ and

$$
x=\frac{Q^{2}}{2 P q}, \quad z=\frac{P P_{h}}{P q}, \quad \cos \theta_{\gamma}=1-\frac{2 M_{N}^{2} x(1-y)}{s y},
$$

where $\theta_{\gamma}$ denotes the angle between target polarization vector and momentum $\mathbf{q}$ of the virtual photon $\gamma^{*}$, see Fig. 2, and $M_{N}$ is the nucleon mass. The component of the momentum of the produced hadron transverse with respect to $\gamma^{*}$ is denoted by $\mathbf{P}_{h \perp}$ and $P_{h \perp}=\left|\mathbf{P}_{h \perp}\right|$.

In the HERMES experiment $A_{U L}^{\sin 2 \phi}$ was measured on proton for pion- [17, 18] and on deuteron target for pionand kaon-production [19] in the kinematic range

$$
\begin{aligned}
& 1 \mathrm{GeV}^{2}<Q^{2}<15 \mathrm{GeV}^{2}, \quad W>2 \mathrm{GeV}, \\
& 0.023<x<0.4, \quad 0.2<y<0.85, \quad 0.2<z<0.7
\end{aligned}
$$

The momenta of produced hadrons were subject to somehow different cuts: $4.5 \mathrm{GeV}<\left|\mathbf{P}_{h}\right|<13.5 \mathrm{GeV}$ in [17, 18] vs. $2 \mathrm{GeV}<\left|\mathbf{P}_{h}\right|<15 \mathrm{GeV}$ in [19]. The resolution cut $P_{h \perp}>50 \mathrm{MeV}$ was applied throughout [17, 18, 19]. This results in the following mean values

$$
\begin{aligned}
& \langle x\rangle=0.09, \quad\langle y\rangle=0.53, \quad\langle z\rangle=0.38 \\
& \left\langle Q^{2}\right\rangle=2.4 \mathrm{GeV}^{2}, \quad\left\langle P_{h \perp}\right\rangle=0.4 \mathrm{GeV} \\
& \langle Q\rangle=1.55 \mathrm{GeV}, \quad\left\langle\cos \theta_{\gamma}\right\rangle=0.98
\end{aligned}
$$

In the experiment the SSA was defined as

$$
A_{U L}^{\sin 2 \phi}=\frac{\sum_{i} \sin \left(2 \phi_{i}\right)\left(N_{i}^{\leftrightarrows}-N_{i}^{\rightrightarrows}\right)}{\sum_{i} \frac{1}{2}\left(N_{i}^{\leftrightarrows}+N_{i}^{\rightrightarrows}\right)}
$$

where $N_{i}^{\leftrightarrows}\left(N_{i}^{\rightrightarrows}\right)$ denotes the number of events $i$ with target polarization antiparallel (parallel) to the beam. 


\section{IV. $A_{U L}^{\sin 2 \phi}$ IN WW-TYPE APPROXIMATION}

The expression for the SSA is given by [7]

$$
A_{U L}^{\sin 2 \phi}(x)=\frac{\int \mathrm{d} y\left[\cos \theta_{\gamma}(1-y) / Q^{4}\right] F_{U L}^{\sin 2 \phi}}{\int \mathrm{d} y\left[\left(1-y+\frac{1}{2} y^{2}\right) / Q^{4}\right] F_{U U, T}}
$$

where in the notation of [37] the numerator is given by

$$
F_{U U, T}(x)=\sum_{a} e_{a}^{2} x f_{1}^{a}(x)\left\langle D_{1}^{a}\right\rangle .
$$

Since our purpose is to test the relation (11), we focus on the $x$-dependence of the SSA, and denote here and in the following averages over $z$ within the cuts (19) by $\langle\ldots\rangle=\int \mathrm{d} z(\ldots)$.

The tree-level expression [7] for the structure function $F_{U L}^{\sin 2 \phi}$ is given in terms of an integral which convolutes transverse parton momenta in the distribution and the fragmentation function (we neglect soft factors [34, 35])

$$
\begin{aligned}
F_{U L}^{\sin 2 \phi}(x, z) & =\int \mathrm{d}^{2} \mathbf{p}_{T} \int \mathrm{d}^{2} \mathbf{K}_{T} \delta^{(2)}\left(z \mathbf{p}_{T}+\mathbf{K}_{T}-\mathbf{P}_{h \perp}\right) \\
& \times\left[\frac{2\left(\mathbf{e}_{h} \mathbf{p}_{T}\right)\left(\mathbf{e}_{h} \mathbf{K}_{T}\right)-\left(\mathbf{p}_{T} \mathbf{K}_{T}\right)}{M_{N} m_{h}}\right] \\
& \times \sum_{a} e_{a}^{2} x h_{1 L}^{\perp a}\left(x, \mathbf{p}_{T}^{2}\right) \frac{H_{1}^{\perp a}\left(z, \mathbf{K}_{T}^{2}\right)}{z}
\end{aligned}
$$

where $\mathbf{e}_{h}=\mathbf{P}_{h \perp} / P_{h \perp}$ and $m_{h}$ denotes the mass of the produced hadron.

Had the events in the numerator of (21) been weighted by $P_{h \perp}^{2} /\left(M_{N} m_{h}\right)$ in addition to $\sin (2 \phi)$, the convolution integral could be solved in a model independent way with the result given in terms of the transverse moment (5) of $h_{1 L}^{\perp}$ and an analog moment for $H_{1}^{\perp}$ [8]. Including such an additional weight makes data analysis more difficult due to acceptance effects. Omitting it, however, forces one to resort to models.

We shall assume the distributions of transverse parton momenta to be Gaussian (and the respective widths $\left\langle\mathbf{p}_{h_{1 L}}^{2}\right\rangle$ and $\left\langle\mathbf{K}_{H_{1}}^{2}\right\rangle$ to be flavour and $x$ - or $z$-independent):

$$
\begin{aligned}
h_{1 L}^{\perp a}\left(x, \mathbf{p}_{T}^{2}\right) & \equiv h_{1 L}^{\perp a}(x) \frac{\exp \left(-\mathbf{p}_{T}^{2} /\left\langle\mathbf{p}_{h_{1 L}}^{2}\right\rangle\right)}{\pi\left\langle\mathbf{p}_{h_{1 L}}^{2}\right\rangle} \\
H_{1}^{\perp a}\left(z, \mathbf{K}_{T}^{2}\right) & \equiv H_{1}^{\perp a}(z) \frac{\exp \left(-\mathbf{K}_{T}^{2} /\left\langle\mathbf{K}_{H_{1}}^{2}\right\rangle\right)}{\pi\left\langle\mathbf{K}_{H_{1}}^{2}\right\rangle}
\end{aligned}
$$

The normalizations are such that one obtains for the unpolarized functions $f_{1}^{a}(x)=\int \mathrm{d}^{2} \mathbf{p}_{T} f_{1}^{a}\left(x, \mathbf{p}_{T}\right)$ and $D_{1}^{a}(z)=\int \mathrm{d}^{2} \mathbf{K}_{T} D_{1}^{a}\left(z, \mathbf{K}_{T}\right)$ with analog Ansätze.

The Gauss Ansatz satisfactorily describes data on many hard reactions [77], provided the transverse momenta are much smaller than the hard scale of the process, i.e. $\left\langle P_{h \perp}\right\rangle \ll\langle Q\rangle$ which is the case at HERMES, see (20). In fact, the $z$-dependence of $\left\langle P_{h \perp}\right\rangle$ at HERMES [19] is well described in the Gauss Ansatz [78].
Of course, one has to keep in mind that (25) is a crude approximation, and it is not clear whether it works also for polarized distribution and fragmentation functions. Moreover, since also unintegrated forms of (3) 4) hold, this Ansatz cannot be equally valid for all pdfs.

What is convenient for our purposes is that (25) allows to solve the convolution integral (24). We obtain

$$
F_{U L}^{\sin 2 \phi}(x)=\sum_{a} e_{a}^{2} x h_{1 L}^{\perp(1) a}(x)\left\langle C_{\text {Gauss }} H_{1}^{\perp(1 / 2) a}\right\rangle .
$$

The $1 / 2$-transverse-moment $H_{1}^{\perp(1 / 2) a}(z)$ and $C_{\text {Gauss }}(z)$, which is also a function of the Gauss model parameters, are defined in App. A. On the basis of the information on the Collins effect from the analyses [54, 55, 56] we estimate

$$
\begin{aligned}
& \left\langle C_{\text {Gauss }} H_{1}^{\perp(1 / 2) \mathrm{fav}}\right\rangle \approx(0.035 \pm 0.008) \times\left(2.2_{-0.1}^{+2.1}\right) \\
& \left\langle C_{\text {Gauss }} H_{1}^{\perp(1 / 2) \mathrm{unf}}\right\rangle \approx-(0.038 \pm 0.007) \times\left(2.2_{-0.1}^{+2.1}\right) .
\end{aligned}
$$

The first factors, with errors due to statistical accuracy of the (preliminary) HERMES data [23], are from [55]. The second factors are due to the transverse momentum dependence of the Collins function; their sizeable uncertainties reflect that the latter is presently poorly constrained by data 56]. See App. A for details.

The errors in (27, 28) are estimated conservatively, such that deviations from our predictions for the SSA should be attributed alone to the failure of (11).

For the estimate of $h_{1 L}^{\perp(1) a}(x)$ by means of (11) we use predictions for the chiral quark-soliton model for $h_{1}^{a}(x)$ 64] as shown in Fig. 17, see Sec. II]

Our results shown in Figs. 3 a-e for pion production from proton and deuteron targets are consistent with the HERMES data 17, 18, 19], and do not exclude that (11) is a useful approximation.

Fig. 3f shows also the SSA for $K^{+}$production. Also here our result is compatible with data [19], however, in this case one tests in addition assumptions on the kaon Collins effect, see App. B.

\section{V. $A_{U L}^{\sin 2 \phi}$ AT CLAS}

One may roughly expect $\left|A_{U L}^{\sin 2 \phi}\right| \lesssim \frac{1}{5}\left|A_{U T}^{\sin \left(\phi-\phi_{S}\right)}\right|$ on the basis of the approximation (11), see App. A. Thus, $A_{U L}^{\sin 2 \phi}$ could be far more difficult to measure than the transverse target Collins effect SSA. Therefore what is needed is a high luminosity experiment sensitive to the region $0.2 \lesssim x \lesssim 0.5$, where the suppression of $h_{1 L}^{\perp(1) a}$ with respect to $h_{1}^{a}(x)$ is less pronounced.

Higher statistics at CLAS at Jefferson Lab, due to two orders of magnitude higher luminosity, provides access to much larger $x$ and larger $z$ than HERMES and COMPASS. Large $z$ may also enhance the SSA due to Collins function $H_{1}^{\perp(1 / 2) a}(z) \propto z D_{1}^{a}(z)$, as observed in [55]. This makes CLAS an ideal experiment for studies of this SSA 

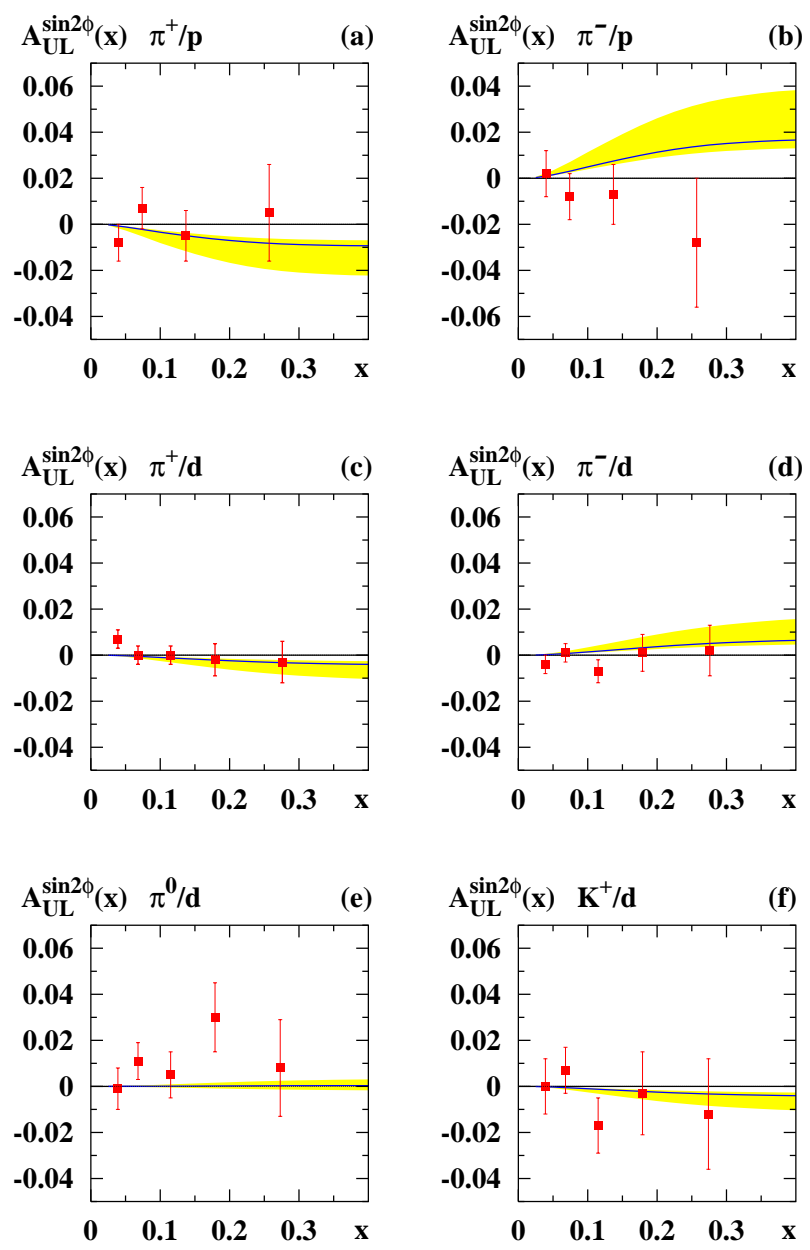

FIG. 3: Longitudinal target SSA $A_{U L}^{\sin 2 \phi}$ as function of $x$. The proton $(\mathrm{a}, \mathrm{b})$ and deuterium $(\mathrm{c}-\mathrm{f})$ target data are from HERMES [17, 19]. The theoretical curves are obtained using information on the Collins fragmentation function from 55 , 56], predictions from the instanton vacuum model and chiral quark soliton model for $h_{L}^{a}(x)$ and $h_{1}^{a}(x)$ [53, 64], and - this is crucial in our context - assuming the validity of the WWtype approximation (11). The shaded error bands are due to the uncertainties in (27, 28), see App. A for details.

in particular and spin-orbit correlations in general. Comparison of the various data sets will also allow to draw valuable conclusions on the energy dependence of the process, possible power-corrections, etc.

The preliminary data from CLAS [25] have shown nonzero SSAs for charged pions, and a compatible with zero within error bars result for $\pi^{0}$. Within our approach it is possible to understand the results for $\pi^{+}$and $\pi^{0}$, however, we obtain for $\pi^{-}$an opposite sign compared to the data. In view of this observation, it is worth to look again on Fig. 3b which shows HERMES data on the $\pi^{-}$-SSA. Does Fig. 3b hint at an incompatibility? Charged pions and in particular the $\pi^{-}$may have significant higher twist contributions, in particular from exclusive vector mesons and semi-exclusive pion production at large $z$.
New data expected from CLAS with $E_{\text {beam }}=6 \mathrm{GeV}$ [79], will increase the existing statistics by about an order of magnitude and more importantly provide comparable to $\pi^{+}$sample of $\pi^{0}$ events. Neutral pion sample is not expected to have any significant contribution from exclusive vector mesons, neither it is expected to have significant higher twist corrections due to semi-exclusive production of pions with large $z$ [80], where the separation between target and current fragmentation is more pronounced.

Higher statistics of upcoming CLAS runs at 6 [79] and $12 \mathrm{GeV}$ [81] will provide access also to higher values of $Q^{2}$ where contributions from exclusive and semi-exclusive processes are more suppressed.

JLab upgrade to $12 \mathrm{GeV}$ will allow to run at an order of magnitude higher luminosities than current CLAS, providing a comprehensive set of single and double spin asymmetries covering a wide range in $x$ and $z$. That will allow detailed studies of kinematic dependences of target SSA and clarify the situation.

\section{VI. $A_{U L}^{\sin 2 \phi}$ AT COMPASS}

COMPASS has taken data with a longitudinally polarized deuterium target which are being analyzed. In near future also a proton target will be used. The $160 \mathrm{GeV}$ muon beam available at COMPASS allows to extend the measurements of $A_{U L}^{\sin 2 \phi}$ and other SSAs into the small $x$-region. By combining all data for $Q^{2}>1 \mathrm{GeV}^{2}$ the average $\left\langle Q^{2}\right\rangle$ at COMPASS is comparable to that at HERMES. Therefore, Figs. 3a-d show roughly our predictions for COMPASS for charged hadron production (at COMPASS about $90 \%$ of the produced charged hadrons are pions).

From (16. 17) one may expect $A_{U L}^{\sin 2 \phi}$ to be substantially smaller, especially at small $x$, than the transverse target SSA $A_{U T}^{\sin \left(\phi+\phi_{S}\right)}$ found compatible with zero in the COMPASS deuterium target experiment [22, 24, 30].

It will be interesting to see whether these predictions will be confirmed by COMPASS.

\section{CONCLUSIONS}

The longitudinal SSA 17, 18, 19, 20 were subject to intensive, early studies [82, 83, 84, 85, 86] that were based on assumptions concerning the flavour dependence of $H_{1}^{\perp}$ [87, 88, 89] that turned out not to be supported by data on Collins effect from SIDIS with transverse target polarization [21, 22, 23, 24] and $e^{+} e^{-}$-annihilations [28, 29]. These data give rise to a new, consistent picture of $H_{1}^{\perp}$ [54, 55, 56] which invites reanalyses of longitudinal SSA.

In this work we did this for $A_{U L}^{\sin 2 \phi} \propto \sum_{a} e_{a}^{2} h_{1 L}^{\perp(1) a} H_{1}^{\perp a}$ from the particular point of view of the question whether there are useful, approximate relations among different TMD pdfs. In fact, QCD equations of motion relate the pdf entering this SSA to $h_{L}^{a}(x)$ and certain pure twist-3 
(and quark mass) terms. Neglecting such terms yields an approximation for $h_{1 L}^{\perp(1) a}$ similar in spirit to the WWapproximation for $g_{T}^{a}(x)$ that is supported by data.

Our study reveals that data do not exclude the possibility that such WW-type approximations work. As a byproduct we observe that data on the two SSAs due to Collins effect, $A_{U L}^{\sin 2 \phi}$ and $A_{U T}^{\sin \left(\phi+\phi_{S}\right)}$, are compatible.

In Ref. [58] predictions for $A_{L T}^{\cos \left(\phi-\phi_{S}\right)} \propto \sum_{a} e_{a}^{2} g_{1 T}^{(1) a} D_{1}^{a}$ were made assuming the validity of a WW-type approximation for the relevant pdf. Comparing these predictions to preliminary COMPASS data 32] one arrives at the same conclusion. Also here data do not exclude the possibility that the WW-type approximation works.

In order to make more definite statements precise measurements of these SSAs are necessary, preferably in the region around $x \sim 0.3$ where the SSAs are largest. An order of magnitude more data on target SSA expected from CLAS upcoming run [79] will certainly improve our current understanding of this and other SSAs and shed light on spin-orbit correlations.

The value of a precise $A_{U L}^{\sin 2 \phi}$ should not be underestimated. This SSA is in any case an independent source of information on the Collins effect. An experimental confirmation of the utility of the WW-type approximation (11), however, would mean that it is possible to extract information on transversity, via (11), from a longitudinally polarized target.

Acknowledgements. We thank M. Anselmino and A. Prokudin for valuable discussions. The work is partially supported by BMBF (Verbundforschung), GermanRussian collaboration (DFG-RFFI) under contract number 436 RUS 113/881/0 and is part of the European Integrated Infrastructure Initiative Hadron Physics project under contract number RII3-CT-2004-506078. A. E. is also supported by the Grants RFBR 06-02-16215 and 07-02-91557, RF MSE RNP.2.2.2.2.6546 (MIREA) and by the Heisenberg-Landau Program of JINR. The work was supported in part by DOE contract DE-AC0506OR23177, under which Jefferson Science Associates, LLC, operates the Jefferson Lab.

\section{APPENDIX A: PION COLLINS EFFECT}

Within the Gauss model one can, of course, rewrite the expression for the SSA (12) in many ways. However, we are interested in exploring the approximation (11) and wish to introduce the transverse moment (5) of $h_{1 L}^{\perp a}$ which in the Gauss model is given by

$$
h_{1 L}^{\perp(1) a}(x) \stackrel{\text { Gauss }}{=} \frac{\left\langle\mathbf{p}_{h_{1 L}}^{2}\right\rangle}{2 M_{N}^{2}} h_{1 L}^{\perp a}(x) .
$$

In order to use information on the Collins function from the analysis of HERMES data [23] in Ref. [55] (the reasons why here this is preferable, are explained in Sec. IV] we introduce the $(1 / 2)$-transverse moment of $H_{1}^{\perp}$ which is defined as and given in Gauss model by

$$
\begin{aligned}
H_{1}^{\perp(1 / 2) a}(z) & \equiv \int \mathrm{d}^{2} \mathbf{K}_{T} \frac{\left|\mathbf{K}_{T}\right|}{2 z m_{\pi}} H_{1}^{\perp a}\left(z, \mathbf{K}_{T}\right) \\
\stackrel{\text { Gauss }}{=} & \frac{\sqrt{\pi}\left\langle\mathbf{K}_{H_{1}}^{2}\right\rangle^{1 / 2}}{4 m_{\pi} z} H_{1}^{\perp a}(z)
\end{aligned}
$$

With the above definitions the numerator of $A_{U L}^{\sin 2 \phi}$ is given by (26) with the function $C_{\text {Gauss }}$ defined as

$$
C_{\text {Gauss }}(z)=\frac{8 z M_{N}}{\left(\pi\left\langle\mathbf{K}_{H_{1}}^{2}\right\rangle\right)^{1 / 2}} \frac{1}{1+z^{2}\left\langle\mathbf{p}_{h_{1 L}}^{2}\right\rangle /\left\langle\mathbf{K}_{H_{1}}^{2}\right\rangle}
$$

In 55] the following information on the Collins effect was obtained from HERMES data [23] on the SSA (14):

$$
\begin{aligned}
& \left\langle 2 B_{\text {Gauss }} H_{1}^{\perp(1 / 2) \text { fav }}\right\rangle=(3.5 \pm 0.8) \%, \\
& \left\langle 2 B_{\text {Gauss }} H_{1}^{\perp(1 / 2) \text { unf }}\right\rangle=-(3.8 \pm 0.7) \%,
\end{aligned}
$$

with

$$
B_{\mathrm{Gauss}}=\frac{1}{\sqrt{1+z^{2}\left\langle\mathbf{p}_{h_{1}}^{2}\right\rangle /\left\langle\mathbf{K}_{H_{1}}^{2}\right\rangle}}
$$

where $\left\langle\mathbf{p}_{h_{1}}^{2}\right\rangle$ is the Gaussian width of the transversity pdf. In order to use the results A4 A5 we approximate

$$
\begin{aligned}
\left\langle C_{\text {Gauss }} H_{1}^{\perp(1 / 2) a}\right\rangle \approx \frac{4\langle z\rangle M_{N}}{\left(\pi\left\langle\mathbf{K}_{H_{1}}^{2}\right\rangle\right)^{1 / 2}} & \underbrace{\left\langle\left\langle 2 B_{\text {Gauss }} H_{1}^{\perp(1 / 2) a}\right\rangle\right.} \frac{2 H_{1}^{\perp(1 / 2) a}}{1+z^{2}\left\langle\mathbf{p}_{h_{1 L}}^{2}\right\rangle /\left\langle\mathbf{K}_{H_{1}}^{2}\right\rangle}\rangle .
\end{aligned}
$$

For $\left\langle\mathbf{K}_{H_{1}}^{2}\right\rangle$ we use results from [56] where Collins function was also assumed to exhibit a Gaussian $k_{T}$-dependence. In the notation of [56] one has

$$
\frac{1}{\left\langle\mathbf{K}_{H_{1}}^{2}\right\rangle}=\frac{1}{\left\langle\mathbf{K}_{D_{1}}^{2}\right\rangle}+\frac{1}{M^{2}}
$$

where the width of the unpolarized fragmentation function was fixed from a study of data on the Cahn effect [90] $\left\langle\mathbf{K}_{D_{1}}^{2}\right\rangle=0.20 \mathrm{GeV}^{2}$. The parameter $M$ was fitted to data from SIDIS and $e^{+} e^{-}$-annihilations (neglecting evolution effects) to be $M^{2}=(0.70 \pm 0.65) \mathrm{GeV}^{2}[56]$. This yields for the first factor in Eq. (A7)

$$
\frac{4 M_{N}\langle z\rangle}{\left(\pi\left\langle\mathbf{K}_{H_{1}}^{2}\right\rangle\right)^{1 / 2}} \simeq 2.2_{-0.1}^{+2.1}
$$

Using for $f_{1}^{a}(x)$ and $D_{1}^{a}(z)$ the LO parameterizations 62 , 91] at $Q^{2}=2.5 \mathrm{GeV}^{2}$ gives the results in Fig. 3.

A remark concerning the error estimates in Fig. 3 is in order. Strictly speaking the errors in (A4, A5 and (A9) are not independent but correlated which we disregard. This means that the errors in Fig. 3 are somehow overestimated. In view of the approximations we make, however, this is not undesired, as it helps to estimate the 
errors more conservatively. With such more conservative error estimates we are on the safe side from the point of view of testing the WW-type approximation (11). In fact, a deviation of our results from data would then presumably be due to a failure of the approximation (11).

We notice the following rough estimate. From (17) and the mean value in (A9) one may estimate roughly

$$
\left|A_{U L}^{\sin 2 \phi}\right| \lesssim \frac{1}{5}\left|A_{U T}^{\sin \left(\phi-\phi_{S}\right)}\right|,
$$

as other factors in the two SSAs are either the same or of similar magnitude.

\section{APPENDIX B: KAON COLLINS EFFECT}

We also wish to estimate the SSA for $K^{+}$. For that we notice that, since pions and kaons are both Goldstone bosons of chiral symmetry breaking, one has in the chiral limit

$$
\lim _{m_{K} \rightarrow 0} \frac{H_{1}^{\perp(1 / 2) a / K}}{D_{1}^{a / K}}=\lim _{m_{\pi} \rightarrow 0} \frac{H_{1}^{\perp(1 / 2) a / \pi}}{D_{1}^{a / \pi}} .
$$

This implies that in the real world with explicit chiral symmetry breaking, i.e. for non-zero pion- and kaonmasses $m_{\pi}$ and $m_{K}$, one may assume the following relations to hold approximately

$$
\frac{H_{1}^{\perp(1 / 2) \bar{s} / K^{+}}}{D_{1}^{\bar{s} / K^{+}}} \approx \frac{H_{1}^{\perp(1 / 2) u / K^{+}}}{D_{1}^{u / K^{+}}} \approx \frac{H_{1}^{\perp(1 / 2) u / \pi^{+}}}{D_{1}^{u / \pi^{+}}}
$$

$$
\frac{H_{1}^{\perp(1 / 2) \mathrm{unf} / K^{+}}}{D_{1}^{\mathrm{unf} / K^{+}}} \approx \frac{H_{1}^{\perp(1 / 2) \mathrm{unf} / \pi^{+}}}{D_{1}^{\mathrm{unf} / \pi^{+}}},
$$

where it is understood that the fragmentation of $d$ - and $\bar{u}$-flavour into $K^{+}$is unfavoured. The estimate (B2) relies on the assumption that "the way from the chiral limit to the real world situation" proceeds quantitatively in a similar way for both polarization dependent and independent quantities. (Notice that the unpolarized "favoured" $\bar{s}$ - and $u$-flavour fragmentations into $K^{+}$are actually different - with the latter being smaller than the former [92]. In the view of the precision of data, however, the effects of strangeness can be neglected due to the smallness of the corresponding pdfs. E.g. the chiral quark soliton model predicts a negligible strangeness contribution to transversity (more precisely: to the tensor charge) [93].)

On the basis of (B1) we estimate

$$
\begin{aligned}
\left\langle 2 B_{\text {Gauss }} H_{1}^{\perp(1 / 2) u / K^{+}}\right\rangle & \approx(1.0 \pm 0.2) \% \\
\left\langle 2 B_{\text {Gauss }} H_{1}^{\perp(1 / 2) \mathrm{unf} / K^{+}}\right\rangle & \approx-(1.0 \pm 0.2) \% .
\end{aligned}
$$

From (B3 B4) we obtain after similar approximations as in App. A the result in Fig. 3:
[1] R. N. Cahn, Phys. Lett. B 78 (1978) 269.

[2] D. W. Sivers, Phys. Rev. D 41, 83 (1990), Phys. Rev. D 43, 261 (1991).

[3] A. V. Efremov, L. Mankiewicz and N. A. Tornqvist, Phys. Lett. B 284 (1992) 394.

[4] J. C. Collins, Nucl. Phys. B 396, 161 (1993) arXiv:hep-ph/9208213.

[5] J. C. Collins, S. F. Heppelmann and G. A. Ladinsky, Nucl. Phys. B 420 (1994) 565 arXiv:hep-ph/9305309.

[6] A. Kotzinian, Nucl. Phys. B 441 (1995) 234 arXiv:hep-ph/9412283.

[7] P. J. Mulders and R. D. Tangerman, Nucl. Phys. B 461 (1996) 197 and 484 (1997) 538E arXiv:hep-ph/9510301.

[8] D. Boer and P. J. Mulders, Phys. Rev. D 57, 5780 (1998) arXiv:hep-ph/9711485.

[9] D. Boer, R. Jakob and P. J. Mulders, Nucl. Phys. B 504 (1997) 345 arXiv:hep-ph/9702281.

[10] D. Boer, R. Jakob and P. J. Mulders, Phys. Lett. B 424 (1998) 143 arXiv:hep-ph/9711488.

[11] D. Boer, Phys. Rev. D 60, 014012 (1999) arXiv:hep-ph/9902255.

[12] S. J. Brodsky, D. S. Hwang and I. Schmidt, Phys. Lett. B 530, 99 (2002) arXiv:hep-ph/0201296; Nucl. Phys. B 642, 344 (2002) arXiv:hep-ph/0206259.
[13] J. C. Collins, Phys. Lett. B 536, 43 (2002) arXiv:hep-ph/0204004.

[14] A. V. Belitsky, X. Ji and F. Yuan, Nucl. Phys. B 656, 165 (2003) arXiv:hep-ph/0208038. X. D. Ji and F. Yuan, Phys. Lett. B 543, 66 (2002) arXiv:hep-ph/0206057]. D. Boer, P. J. Mulders and F. Pijlman, Nucl. Phys. B 667, 201 (2003) arXiv:hep-ph/0303034.

[15] For a discussion and careful definition of transverse momentum dependent distribution functions in QCD we refer to: J. C. Collins, Acta Phys. Polon. B 34, 3103 (2003) arXiv:hep-ph/0304122.

[16] M. Arneodo et al. [European Muon Collaboration], Z. Phys. C 34 (1987) 277.

[17] A. Airapetian et al. [HERMES Collaboration], Phys. Rev. Lett. 84, 4047 (2000) arXiv:hep-ex/9910062. H. Avakian [HERMES Collaboration], Nucl. Phys. Proc. Suppl. 79, 523 (1999).

[18] A. Airapetian et al. [HERMES Collaboration], Phys. Rev. D 64, 097101 (2001) arXiv:hep-ex/0104005.

[19] A. Airapetian et al. [HERMES Collaboration], Phys. Lett. B 562, 182 (2003) arXiv:hep-ex/0212039.

[20] H. Avakian et al. [CLAS Collaboration], Phys. Rev. D 69, 112004 (2004) arXiv:hep-ex/0301005.

[21] A. Airapetian et al. [HERMES Collaboration], Phys. 
Rev. Lett. 94, 012002 (2005) arXiv:hep-ex/0408013.

[22] V. Y. Alexakhin et al. [COMPASS Collaboration], Phys. Rev. Lett. 94, 202002 (2005) arXiv:hep-ex/0503002.

[23] M. Diefenthaler, AIP Conf. Proc. 792 (2005) 933 arXiv:hep-ex/0507013.

I. M. Gregor [HERMES Collaboration], Acta Phys. Polon. B 36, 209 (2005).

[24] E. S. Ageev et al. [COMPASS Collaboration], Nucl. Phys. B 765 (2007) 31 arXiv:hep-ex/0610068.

[25] H. Avakian, P. Bosted, V. Burkert and L. Elouadrhiri [CLAS Collaboration], AIP Conf. Proc. 792 (2005) 945 arXiv:nucl-ex/0509032.

[26] A. Airapetian et al. [HERMES Collaboration], Phys. Lett. B 622, 14 (2005) arXiv:hep-ex/0505042.

[27] A. Airapetian et al. [HERMES Collaboration], Phys. Lett. B 648 (2007) 164 arXiv:hep-ex/0612059.

[28] K. Abe et al. [Belle Collaboration], Phys. Rev. Lett. 96, 232002 (2006) arXiv:hep-ex/0507063.

[29] A. Ogawa, M. Grosse-Perdekamp, R. Seidl and K. Hasuko, arXiv:hep-ex/0607014.

[30] A. Martin [COMPASS Collaboration], Czech. J. Phys. 56 (2006) F33 arXiv:hep-ex/0702002.

[31] M. Diefenthaler [HERMES Collaboration], arXiv:0706.2242 [hep-ex], and arXiv:hep-ex/0612010.

[32] A. Kotzinian [on behalf of the COMPASS collaboration], arXiv:0705.2402 [hep-ex].

[33] J. C. Collins and D. E. Soper, Nucl. Phys. B 193, 381 (1981) [Erratum-ibid. B 213, 545 (1983)].

[34] X. D. Ji, J. P. Ma and F. Yuan, Phys. Rev. D 71, 034005 (2005) arXiv:hep-ph/0404183, Phys. Lett. B 597, 299 (2004) arXiv:hep-ph/0405085.

[35] J. C. Collins and A. Metz, Phys. Rev. Lett. 93, 252001 (2004) arXiv:hep-ph/0408249.

[36] K. Goeke, A. Metz and M. Schlegel, Phys. Lett. B 618, 90 (2005) arXiv:hep-ph/0504130.

[37] For a review see: A. Bacchetta, M. Diehl, K. Goeke, A. Metz, P. J. Mulders and M. Schlegel, JHEP 0702 (2007) 093 arXiv:hep-ph/0611265.

[38] J. P. Ralston and D. E. Soper, Nucl. Phys. B 152 (1979) 109.

[39] R. L. Jaffe and X. D. Ji, Nucl. Phys. B 375 (1992) 527, and Phys. Rev. Lett. 67, 552 (1991).

[40] S. Wandzura and F. Wilczek, Phys. Lett. B 72 (1977) 195.

[41] For completeness we note that in the analog decompostion for $e^{a}(x)$ one encounters, apart from a $\delta$-function singularity at $x=0$, pure twist- 3 and mass-terms only, see A. V. Efremov and P. Schweitzer, JHEP 0308 (2003) 006 arXiv:hep-ph/0212044 for a review.

[42] E. V. Shuryak and A. I. Vainshtein, Nucl. Phys. B 201 (1982) 141.

[43] For a review on $g_{2}^{a}(x) \equiv g_{T}^{a}(x)-g_{1}^{a}(x)$ see: R. L. Jaffe, Comments Nucl. Part. Phys. 19 (1990) 239.

[44] R. L. Jaffe, in "The spin structure of the nucleon", edited by B. Frois, V.W. Hughes, N. de Groot (Singapore, World Scientific, 1997), p. 42 arXiv:hep-ph/9602236.

[45] X. Zheng et al. [Jefferson Lab Hall A Collaboration], Phys. Rev. C 70, 065207 (2004) arXiv:nucl-ex/0405006.

[46] M. Amarian et al. [Jefferson Lab E94-010 Collaboration], Phys. Rev. Lett. 92 (2004) 022301 arXiv:hep-ex/0310003.

[47] P. L. Anthony et al. [E155 Collaboration], Phys. Lett. B 553 (2003) 18 arXiv:hep-ex/0204028.

[48] K. Abe et al. [E143 collaboration], Phys. Rev. D 58
(1998) 112003 arXiv:hep-ph/9802357.

[49] D. Adams et al. [Spin Muon Collaboration (SMC)], Phys. Lett. B 336 (1994) 125 arXiv:hep-ex/9408001.

[50] M. Gockeler et al., Phys. Rev. D 63 (2001) 074506 arXiv:hep-lat/0011091.

[51] M. Gockeler et al., Phys. Rev. D 72 (2005) 054507 arXiv:hep-lat/0506017.

[52] J. Balla, M. V. Polyakov and C. Weiss, Nucl. Phys. B 510 (1998) 327 arXiv:hep-ph/9707515.

[53] B. Dressler and M. V. Polyakov, Phys. Rev. D 61 (2000) 097501 arXiv:hep-ph/9912376].

[54] W. Vogelsang and F. Yuan, Phys. Rev. D 72 (2005) 054028 arXiv:hep-ph/0507266.

[55] A. V. Efremov, K. Goeke and P. Schweitzer, Phys. Rev. D 73, 094025 (2006) arXiv:hep-ph/0603054.

[56] M. Anselmino, M. Boglione, U. D'Alesio, A. Kotzinian, F. Murgia, A. Prokudin and C. Turk, Phys. Rev. D 75, 054032 (2007) arXiv:hep-ph/0701006.

[57] L. P. Gamberg, G. R. Goldstein and M. Schlegel, arXiv:0708.2580 [hep-ph] and arXiv:0708.0324 [hep-ph].

[58] A. Kotzinian, B. Parsamyan and A. Prokudin, Phys. Rev. D 73, 114017 (2006) arXiv:hep-ph/0603194.

[59] A. M. Kotzinian and P. J. Mulders, Phys. Rev. D 54 (1996) 1229 arXiv:hep-ph/9511420.

[60] H. Avakian, S. J. Brodsky, A. Deur and F. Yuan, arXiv:0705.1553 [hep-ph].

[61] J. Soffer, Phys. Rev. Lett. $74 \quad$ (1995) 1292 arXiv:hep-ph/9409254.

[62] M. Glück, E. Reya and A. Vogt, Eur. Phys. J. C 5, 461 (1998) arXiv:hep-ph/9806404.

[63] M. Glück, E. Reya, M. Stratmann and W. Vogelsang, Phys. Rev. D 63, 094005 (2001) arXiv:hep-ph/0011215.

[64] P. Schweitzer, D. Urbano, M. V. Polyakov, C. Weiss, P. V. Pobylitsa and K. Goeke, Phys. Rev. D 64 (2001) 034013 arXiv:hep-ph/0101300. P. V. Pobylitsa and M. V. Polyakov, Phys. Lett. B 389, 350 (1996) arXiv:hep-ph/9608434.

[65] B. Pasquini, M. Pincetti and S. Boffi, Phys. Rev. D 76 (2007) 034020 arXiv:hep-ph/0612094.

[66] A. V. Efremov, O. V. Teryaev and P. Zavada, Phys. Rev. D 70 (2004) 054018 arXiv:hep-ph/0405225.

[67] For a review on transversity, see: V. Barone, A. Drago and P. G. Ratcliffe, Phys. Rept. 359 (2002) 1 arXiv:hep-ph/0104283.

[68] S. J. Brodsky and F. Yuan, Phys. Rev. D 74 (2006) 094018 arXiv:hep-ph/0610236.

[69] X. Artru and M. Mekhfi, Z. Phys. C 45 (1990) 669.

[70] W. Vogelsang, Phys. Rev. D 57, 1886 (1998) arXiv:hep-ph/9706511.

A. Hayashigaki, Y. Kanazawa and Y. Koike, Phys. Rev. D 56, 7350 (1997) arXiv:hep-ph/9707208.

S. Kumano and M. Miyama, Phys. Rev. D 56, 2504 (1997) arXiv:hep-ph/9706420.

[71] R. Kirschner, L. Mankiewicz, A. Schäfer and $\begin{array}{lllllll}\text { L. Szymanowski, Z. Phys. C } & \mathbf{7 4} & \text { (1997) } & 501\end{array}$ arXiv:hep-ph/9606267.

[72] D. Diakonov, V. Y. Petrov and P. V. Pobylitsa, Nucl. Phys. B 306, 809 (1988).

[73] C. V. Christov et al., Prog. Part. Nucl. Phys. 37 (1996) 91 arXiv:hep-ph/9604441].

[74] D. Diakonov et al., Nucl. Phys. B 480, 341 (1996) arXiv:hep-ph/9606314, Phys. Rev. D 56, 4069 (1997) arXiv:hep-ph/9703420, P. V. Pobylitsa et al., Phys. Rev. D 59, 034024 (1999) arXiv:hep-ph/9804436. 
M. Wakamatsu and T. Kubota, Phys. Rev. D 60, 034020 (1999) arXiv:hep-ph/9809443. K. Goeke et al., Acta Phys. Polon. B 32, 1201 (2001) arXiv:hep-ph/0001272.

[75] D. Diakonov and V. Y. Petrov, Nucl. Phys. B 245 (1984) 259; Nucl. Phys. B 272, 457 (1986).

[76] D. Diakonov, M. V. Polyakov and C. Weiss, Nucl. Phys. B 461, 539 (1996) arXiv:hep-ph/9510232.

[77] U. D'Alesio and F. Murgia, Phys. Rev. D 70, 074009 (2004) arXiv:hep-ph/0408092.

[78] J. C. Collins, A. V. Efremov, K. Goeke, S. Menzel, A. Metz and P. Schweitzer, Phys. Rev. D 73 (2006) 014021 arXiv:hep-ph/0509076.

[79] H. Avakian et al, JLab E05-113, "Semi-Inclusive Pion Production with a Longitudinally Polarized Target at 6GeV", JLab proposal.

[80] A. Afanasev, C. E. Carlson and C. Wahlquist, Phys. Lett. B 398 (1997) 393 arXiv:hep-ph/9701215.

[81] H. Avakian et al, JLab PR12-07-107, "Studies of SpinOrbit Correlations with Longitudinally Polarized Target", JLab proposal.

[82] E. De Sanctis, W. D. Nowak and K. A. Oganessian, Phys. Lett. B 483, 69 (2000) arXiv:hep-ph/0002091.

K. A. Oganessian, N. Bianchi, E. De Sanctis and W. D. Nowak, Nucl. Phys. A 689, 784 (2001) arXiv:hep-ph/0010261.

[83] M. Anselmino and F. Murgia, Phys. Lett. B 483 (2000) 74 arXiv:hep-ph/0002120.

[84] A. V. Efremov, K. Goeke and P. Schweitzer, Phys. Lett. B 522, 37 (2001) arXiv:hep-ph/0108213 and 544, 389E
(2002) arXiv:hep-ph/0204056; Acta Phys. Polon. B 33, 3755 (2002) arXiv:hep-ph/0206267.

[85] A. V. Efremov, K. Goeke and P. Schweitzer, Eur. Phys. J. C 24 (2002) 407 arXiv:hep-ph/0112166; Phys. Lett. B 568 (2003) 63 arXiv:hep-ph/0303062.

[86] B. Q. Ma, I. Schmidt and J. J. Yang, Phys. Rev. D 66, 094001 (2002) arXiv:hep-ph/0209114]; Phys. Rev. D 65, 034010 (2002) arXiv:hep-ph/0110324.

[87] A. Bacchetta, R. Kundu, A. Metz and P. J. Mulders, Phys. Rev. D 65, 094021 (2002) arXiv:hep-ph/0201091.

[88] A. V. Efremov, K. Goeke and P. Schweitzer, Eur. Phys. J. C 32 (2003) 337 arXiv:hep-ph/0309209. P. Schweitzer and A. Bacchetta, Nucl. Phys. A 732, 106 (2004) arXiv:hep-ph/0310318.

[89] For reviews see: A. V. Efremov, Annalen Phys. 13 (2004) 651 arXiv:hep-ph/0410389.

A. V. Efremov, K. Goeke and P. Schweitzer, Czech. J. Phys. 55 (2005) A189 arXiv:hep-ph/0412420.

[90] M. Anselmino, M. Boglione, U. D'Alesio, A. Kotzinian, F. Murgia and A. Prokudin, Phys. Rev. D 71 (2005) 074006 arXiv:hep-ph/0501196].

[91] S. Kretzer, E. Leader and E. Christova, Eur. Phys. J. C 22, 269 (2001) arXiv:hep-ph/0108055.

[92] S. Kretzer, Phys. Rev. D $62 \quad$ (2000) 054001 arXiv:hep-ph/0003177.

[93] H. C. Kim, M. V. Polyakov and K. Goeke, Phys. Lett. B 387, 577 (1996) arXiv:hep-ph/9604442. 